\title{
The association between inflammation, the microbiome and urethane-induced pulmonary adenocarcinoma
}

\author{
ZENGHUA DENG ${ }^{1}$, ZHIHUI LI ${ }^{2}$, CHANGQING SUN ${ }^{1}$, HUI XIE ${ }^{1}$, ZHENGANG CHEN ${ }^{1}$, \\ JINBO LIU ${ }^{1}$, HUI WANG ${ }^{1}$, CHENGGANG ZHANG $^{2}$ and GUANGSHUN WANG ${ }^{1}$ \\ ${ }^{1}$ Baodi Clinical Institute, Tianjin Medical University, Tianjin 301800; ${ }^{2}$ Beijing Institute of Radiation Medicine, \\ State Key Laboratory of Proteomics, Cognitive and Mental Health Research Center of The PLA, Beijing 100850, P.R. China
}

Received April 9, 2016; Accepted December 20, 2016

DOI: $10.3892 / \mathrm{ol} .2018 .8167$

\begin{abstract}
Lung cancer is amongst the most common types of cancer throughout the world. The overall 5-year survival rate is $\sim 17 \%$. A number of studies have demonstrated that the microbiome existing within the host may affect the level of inflammation, and consequently contribute to the carcinogenesis of certain types of cancer. To investigate the role of inflammation and the microbiome in the carcinogenesis of lung cancer, an intervention study involving mice, including a control group $(C ; n=5)$, a urethane-induced pulmonary adenocarcinoma group $(\mathrm{U} ; \mathrm{n}=5)$ and a prebiotics intervention group $(\mathrm{P} ; \mathrm{n}=5)$ was carried out. This pulmonary adenocarcinoma model was reviewed, and incidences of the disease were identified using histopathology. The levels of the inflammatory cytokines nuclear factor $\kappa \mathrm{B}(\mathrm{NF}-\kappa \mathrm{B})$, tumor necrosis factor $\alpha(\mathrm{TNF}-\alpha)$, interleukin-1 $\beta$ (IL-1 $\beta$ ) and IL-6 in the sera samples were measured using an ELISA technique. In addition, high-throughput sequencing of the $16 \mathrm{~S}$ ribosomal RNA gene segment was used to analyze the species present in the microbiome of the lower airways and intestinal tracts of mice. The results demonstrated that groups $\mathrm{P}$ and $\mathrm{U}$ exhibited altered histopathology and the development of lung adenocarcinoma tumors, but no differences were observed between the groups. The level of inflammation, determined by measuring the levels of NF- $\kappa$ B, TNF- $\alpha$, IL-1 $\beta$ and IL-6 inflammatory cytokines, was significantly lower in group $\mathrm{P}$ compared with group $\mathrm{U}$ $(\mathrm{P}<0.05)$, and was significantly higher in group $\mathrm{P}$ compared
\end{abstract}

Correspondence to: Professor Guangshun Wang, Baodi Clinical Institute, Tianjin Medical University, 8 Guangchuan Road, Baodi, Tianjin 301800, P.R. China

E-mail:wgs@bddhospital.com

Professor Chenggang Zhang, Beijing Institute of Radiation Medicine, State Key Laboratory of Proteomics, Cognitive and Mental Health Research Center of the PLA, 27 Taiping Road, Haidian, Beijing 100850, P.R. China

E-mail: zcgweb@126.com

Keywords: inflammation, microbiome, pulmonary adenocarcinoma, urethane, prebiotics with group $\mathrm{C}(\mathrm{P}<0.05)$. Overall, the microbiomes of the lower respiratory and intestinal tracts did not change markedly among the 3 groups, in terms of the size of colonies and Shannon diversity indices. However, at a family and operational taxonomic unit (OTU) level, certain microbiota were altered. For example, the abundance of the Clostridiales and Lachnospiraceae families was lower in the lung and intestinal tracts subsequent to urethane-induced treatment compared with in the control group $(\mathrm{P}<0.05)$, and the level of abundance of the Clostridiales family increased to similar levels within the control group $(\mathrm{P}<0.05)$, when prebiotics were administered. The levels of abundance of the S24-7, Bacteroidales and Firmicutes families were higher in the intestinal tract compared with the control group $(\mathrm{P}<0.05)$, and following treatment with prebiotics, the levels of abundance of these families decreased to similar levels observed in the control group $(\mathrm{P}<0.05)$. In conclusion, inflammation and the microbiome serve important roles in the carcinogenesis of lung cancer. Additionally, prebiotics may increase the efficacy of lung cancer treatment by modulating levels of inflammation and the composition of the microbiome. The associations between inflammation, the microbiome and lung cancer require attention.

\section{Introduction}

Lung cancer is the leading cause of cancer mortality amongst males globally, and has surpassed breast cancer as the leading cause of cancer mortality amongst females in developed countries (1). The 5-year survival rate has remained poor, at $\sim 17 \%$, over previous decades despite multiple novel aggressive therapies (2). Furthermore, the majority of patients are diagnosed whilst in the advanced stages of the disease, due to a lack of clinical presentation within the early stages. Thus, novel targets and approaches are required to improve the treatment and diagnosis of lung cancer.

Recently, the human microbiome has been an active area of research. A previous study demonstrated that the human intestine contains $\sim 100$ trillion microorganisms, including 500-1,000 different species (3). Although the gastrointestinal tract is the major colony site for these microorganisms, other sites of colonization include the oral cavity, the respiratory tract, the urogenital tract and the skin. At present, these complex microbiomes remain incompletely characterized. 
However, many studies have focused on the association between the microbiome and cancer, including Helicobacter pylori and gastric carcinoma (4), Epstein-Barr virus and nasopharyngeal carcinoma (5), and Human papillomavirus and cervical cancer (6). The majority of the earlier studies focus on a single pathogenic bacteria or virus.

Developments in metagenomics including $16 \mathrm{~S}$ rRNA gene pyrosequencing techniques and bioinformatics (7) have assisted in providing an understanding of the role of the human microbiome within cancer. In the oral cavity, changes in the level of microorganisms are considered to be associated with the development of oral cancer (8). In the pancreatic cancer, the levels of Neisseria elongata and Streptococcus mitis in the saliva have been revealed to be increased in patients with pancreatic cancer, and may become the microbial biomarkers for pancreatic cancer (9). In the colon, certain bacterial biofilms were associated with colorectal cancer, and fecal microbiota may be a potential target for early-stage detection methods for colorectal cancer $(10,11)$. In the respiratory tract, microbiota is associated with many lung diseases, including chronic obstructive pulmonary disease (COPD) $(12,13)$, asthma (14) and lung cancer (15). These studies demonstrated that the human microbiome is associated with cancer.

Numerous studies have indicated that inflammation is an important factor in the promotion of carcinogenesis in pulmonary adenocarcinoma $(16,17)$. Additionally, recent studies revealed that the microbiome serves an important role in the regulation of inflammation through numerous mechanisms, such as influencing the metabolism of short-chain fatty acids, which were recognized to be beneficial to the host. Furthermore, the level of inflammation of the host may be reduced when imbalances within the microbiome are reversed $(18,19)$. A previous epidemiological study demonstrated that $\sim 16 \%$ of types of human cancer worldwide were associated with infectious agents or infection-associated chronic inflammation, with a higher percentage in developing countries, at $22.9 \%$, compared with developed countries, at $7.4 \%$ (20).

Understanding the role of the microbiome in lung cancer is important to identify novel targets and approaches for the treatment and diagnosis of the disease. In the present study, a urethane-induced pulmonary adenocarcinoma model was used to explore the roles of the microbiomes in the lower respiratory and intestinal tracts, and of inflammation, determined by measuring the levels of inflammatory cytokines nuclear factor $\kappa \mathrm{B}(\mathrm{NF}-\kappa \mathrm{B})$, tumor necrosis factor $\alpha$ (TNF- $\alpha$ ), interleukin-1 $\beta$ (IL-1 $\beta$ ) and IL-6 present in lung adenocarcinoma. Additionally, prebiotics were used to alter the microbiome and levels of inflammation in urethane-induced pulmonary adenocarcinoma. The result revealed that inflammation and the microbiome may serve an important role in the carcinogenesis of lung cancer, and that prebiotics may assist the function of treatments of lung cancer by modulating the microbiome and the inflammatory response.

\section{Materials and methods}

Animals, groups and sample collection. A total of 15 6-week-old male BALB/c mice, weighing 18-22 g, were sourced from Vital River Laboratories Co., Ltd., (Beijing, China), and were randomly distributed into 3 groups with access to tap water and an unrestricted diet. Each group included 5 animals in 1 cage. The environment was maintained at $19-22^{\circ} \mathrm{C}$, with $40-60 \%$ humidity and a standard $12 \mathrm{~h}$ day/night rhythm. The animals were given 2 weeks to adapt to the novel environment.

All experiments (performed once) included: A control group (C); a urethane-induced adenocarcinoma group (U) and a prebiotics-gavage intervention group $(\mathrm{P})$. The cases of pulmonary adenocarcinoma were induced by intraperitoneal (i.p.) injection of $1 \mathrm{~g} / \mathrm{kg}$ dose of urethane in $100 \mu \mathrm{l}$ saline once per week for 8 weeks. The control group was injected i.p. with $100 \mu$ l saline, as described previously $(21,22)$. Group $\mathrm{P}$ was treated intragastrically with $15 \mathrm{mg} / \mathrm{kg}$ prebiotics 5 times/week continuously for 16 weeks, beginning at the 5th week subsequent to the first injection of the urethane, whilst groups $\mathrm{C}$ and $\mathrm{U}$ were treated with equivalent saline. The prebiotics in this experiment were: Dietary fiber (extracted from endive and konjak), Cordyceps, Ginseng, Ganoderma lucidum, Seaweed, Hericium and Lycium barbarum polysaccharides.

All of the mice were sacrificed (via exsanguination following anesthesia with 5\% isoflurane) at the end of the 20th week after the first injection of urethane. Fresh stool and sera samples were collected and stored at $-80^{\circ} \mathrm{C}$ prior to sacrifice. The bronchoalveolar lavage (BAL) procedure was performed, as described in previous studies $(22,23)$. All BAL samples were stored at $-80^{\circ} \mathrm{C}$, and the lung tissue was fixed in $10 \%$ paraformaldehyde for histopathological analyses.

Serum inflammation measurements. NF- $\kappa$ B, TNF- $\alpha$, IL-1 $\beta$ and IL-6 levels in serum were detected through a mouse $(\mathrm{NF}-\kappa \mathrm{B}, \mathrm{TNF}-\alpha, \mathrm{IL}-1 \beta$ and IL-6) ELISA kit (Elabscience Biotechnology Co., Ltd., Wuhan, China). The optical density was read at $450 \mathrm{~nm}$ using a microtiter plate reader.

DNA extraction. Extraction of the DNA from 180-220 mg of the fresh stool samples using the TIANamp Stool DNA kit (DP328; TIANGEN Biotech Co., Ltd., Beijing, China) was carried out according to protocol of the manufacturer. However, $400 \mathrm{~g}$ of the BAL samples were first thawed and centrifuged for $5 \mathrm{~min}$ at room temperature and $400 \mathrm{x} \mathrm{g}$, and the supernatant was collected. The supernatant samples were then centrifuged at $13,210 \mathrm{x}$ g for $5 \mathrm{~min}$ at room temperature again. Subsequently, the bacterial DNA from the BAL samples was extracted using the TIANamp Micro DNA kit (DP316; TIANGEN Biotech Co., Ltd.) according to protocol outlined previously (23). All DNA quality was assessed using gel electrophoresis and spectrophotometry, and was stored at $-80^{\circ} \mathrm{C}$ prior to use.

$16 S$ sequencing and bioinformatics. 16S rRNA (V4) sequencing was carried out to analyze the changes in the microbiome in accordance with protocol described in a previous study (24). The sequences were quality-checked and clustered into de novo operational taxonomic units (OTUs) at the $97 \%$ similarity threshold for the production of OTUs using UPARSE software (25, http://drive5.com/uparse/). The most abundant sequence in each OTU was chosen as the representative sequence. UCHIME software was used to remove chimeric sequences (26). Outliers with a low sequence count and microbial diversity were removed. Mothur software (version 1.35.1; 
https://www.mothur.org/) was then used to calculate indices of Shannon diversity and richness (27). The Ribosomal Database Project (RDP) Classifier software (version 16; https://rdp.cme. msu.edu/) was used to classify sequences.

Statistical analysis. The data was analyzed using a 2-sample t-test and SPSS 14.0 software (SPSS, Inc., Chicago, IL, USA). $\mathrm{P}<0.05$ was considered to indicate a statistically significant difference. Two sample t-tests were performed to identify significant differences between families and OTUs between each group, and data are presented as the mean \pm standard deviation.

\section{Results}

Constructing and identifying the model of lung cancer. As demonstrated in Fig. 1, at the end of the 20th week, subsequent to the first injection of urethane, each mouse in groups $\mathrm{U}$ and $\mathrm{P}$ displayed altered histopathology and the development of lung adenocarcinoma tumors, whilst the control group did not.

Prebiotics inhibited urethane-induced elevation of the level of inflammation $(N F-\kappa B, T N F-\alpha, I L-1 \beta, I L-6)$ in serum. The levels of the inflammatory markers NF- $\kappa$ B, TNF- $\alpha$, IL- $1 \beta$ and IL- 6 were markedly higher in groups $U$ and $P$ than in the control group after urethane-induced treatment, as illustrated in Fig. 2. When the urethane-induced adenocarcinoma mice were treated with prebiotics, the levels of these inflammatory markers markedly decreased to similar levels observed within the control.

Richness and diversity of microbiome in lung and intestine of mice. To investigate the change in the composition of the microbiome, the bacterial richness and Shannon diversity indices of each group were calculated and compared for the lung tissues, with samples obtained from BAL, and for the intestinal tissues, with samples obtained from feces. The results are demonstrated in Fig. 3A-D.

In the lower airways, the mean richness values were $570.6 \pm 194.2,594.2 \pm 250.4,583.4 \pm 160.0$ for groups C, U and $\mathrm{P}$, respectively, and were not statistically significant as illustrated in (Fig. 3A) (U vs. C; $\mathrm{P}=0.8719$; $\mathrm{P}$ vs. $\mathrm{C}, \mathrm{P}=0.9122$; $\mathrm{P}$ vs. $\mathrm{U}, \mathrm{P}=0.9372)$. In the intestinal tract, the mean richness values which were $515.4 \pm 51.8,510.4 \pm 81.8,512.4 \pm 41.3$ for groups $\mathrm{C}, \mathrm{U}$ and $\mathrm{P}$, respectively, and were also not significantly different, as demonstrated in Fig. 3B (U vs. C; $\mathrm{P}=0.9109$; $\mathrm{P}$ vs. $\mathrm{C}, \mathrm{P}=0.9218$; $\mathrm{P}$ vs. $\mathrm{U}, \mathrm{P}=0.9623$ ). In the aforementioned 3 groups, differences in the Shannon diversity indices were not statistically significant in the lower respiratory or the intestinal tracts, as demonstrated in Fig. 3C (U vs. C; $\mathrm{P}=0.9978$; $\mathrm{P}$ vs. C, $\mathrm{P}=0.7978$; $\mathrm{P}$ vs. $\mathrm{U} ; \mathrm{P}=0.8837$ ) and Fig. $3 \mathrm{D}$ (U vs. $\mathrm{C} ; \mathrm{P}=0.4056$; $\mathrm{P}$ vs. $\mathrm{C}, \mathrm{P}=0.5106$; $\mathrm{P}$ vs. $\mathrm{U}, \mathrm{P}=0.6586)$.

Composition of microbial communities at the class level. A total of 30 classes of bacteria were identified using the RDP classifier. Gammaproteobacteria, Chloroplasts, Alphaproteobacteria, Bacteroidia, Bacilli, Clostridia and Betaproteobacteria were the most abundant classes in BAL, and occupied $94.9 \%$ of the microbiota in the $3 \mathrm{BC}, \mathrm{BU}$ and

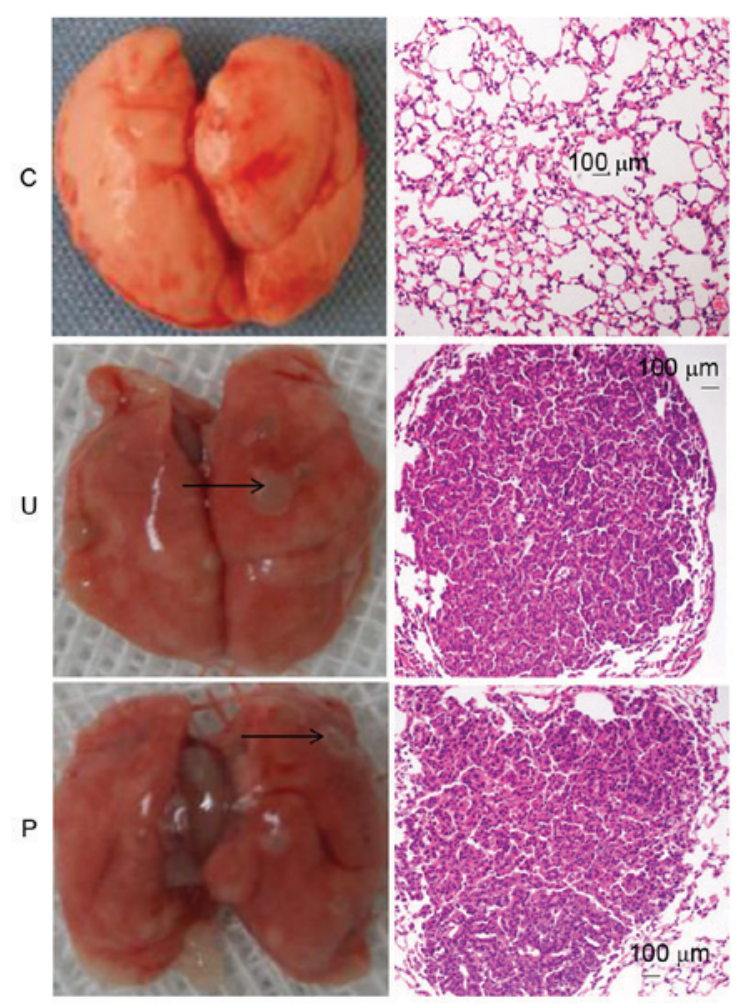

Figure 1. Differences in pathology between each group. The right column is hematoxylin and eosin staining $(\mathrm{d}=5 \mu \mathrm{m})$ results from each group, and the left column is the lung tissue from each group. Arrows denote lesions on the lung surface. At the end of the 20th week, subsequent to the first injection of urethane, each mouse in groups $\mathrm{U}$ and $\mathrm{P}$ exhibited altered histopathology and the development of lung adenocarcinoma tumors, whilst the control group did not. C, control group; U, urethane-induced adenocarcinoma group; $\mathrm{P}$, prebiotics-gavage intervention group.

BP groups, as illustrated in Fig. 4. No significant difference was observed in the types of classes of bacteria between the groups $\mathrm{C}, \mathrm{P}$ and $\mathrm{U}$ in the lower respiratory tract microbiome. In the intestinal tract, Clostridia and Bacteroidia were the most abundant types of class, and occupied $88.99 \%$ of the microbiota in the 3 (FC, FU and FP) groups. No significant variation in the types of classes of bacteria was observed between the 3 groups in these samples.

Composition of microbial communities at the family level. To analyze the systematic differences in microbial communities among groups C, U and P, 68 families except the 'Others' group, comprising other types of microbes whose total ratio was $<0.5 \%$, were clustered based on their respective relative abundance, as demonstrated in Fig. 5. In the lower respiratory tract microbiome, no significant difference was identified between groups $\mathrm{C}, \mathrm{U}$ and $\mathrm{P}$. In the intestinal tract, the levels of abundance of the Oxalobacteraceae and Paenibacillaceae families were lower in group $\mathrm{U}$ compared with group $\mathrm{C}$. The levels of abundance of these families were higher in group P compared with group $\mathrm{U}$, although the difference was not statistically significant. The levels of abundance of the Enterobacteriaceae, Moraxellaceae, and Shewanellaceae families significantly increased in group P compared with group U. Additionally, all abundance levels of these families were lower in group $\mathrm{U}$ compared with group $\mathrm{C}$, although the difference 

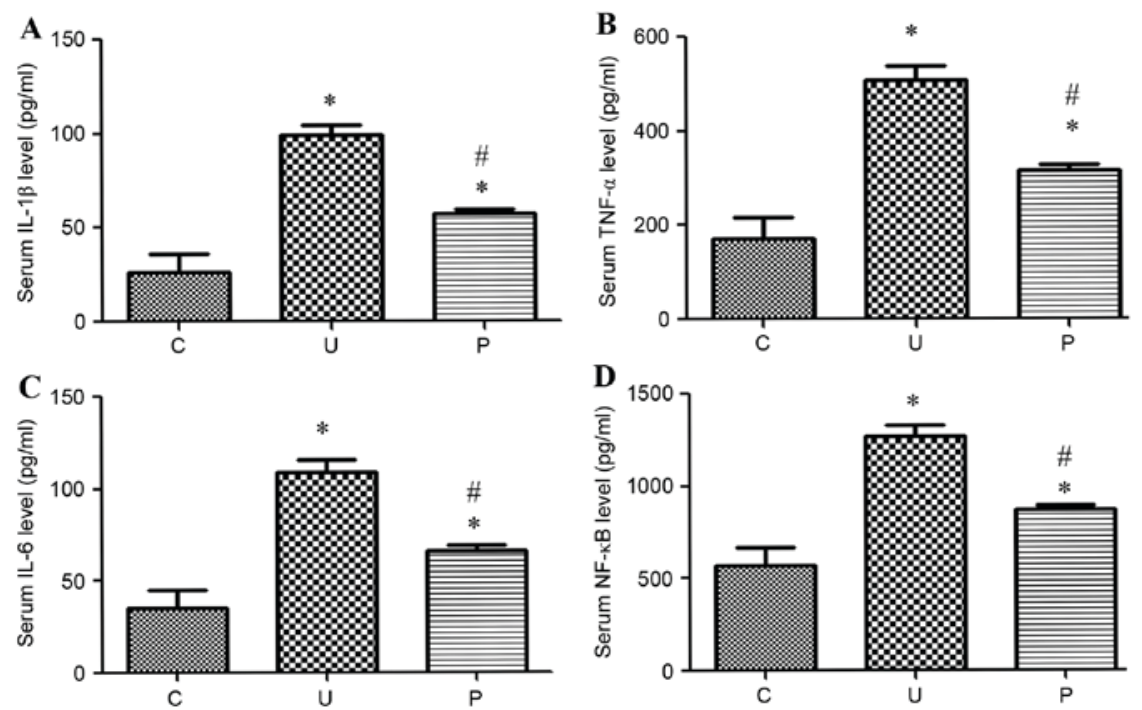

Figure 2. Levels of inflammation ( $\mathrm{pg} / \mathrm{ml}$ ) in sera in each group ( $\mathrm{n}=5$ for each group). The levels of inflammatory markers (A) IL-1 $\beta$, (B) TNF- $\alpha$, (C) IL-6 and (D) NF-kB were significantly higher in both groups $\mathrm{U}(\mathrm{P}<0.0001,<0.0001,<0.0001$ and $<0.0001$ respectively) and $\mathrm{P}(\mathrm{P}<0.0001,<0.0002,<0.0001$ and 0.0002 respectively) than in the control group $\mathrm{C}$. The level of inflammatory markers was significantly decreased in group P compared with group $\mathrm{U}$ $\left(\mathrm{P}<0.0001,<0.0001,<0.0001\right.$ and $<0.0001$ respectively). Data are presented as the mean \pm standard deviation. ${ }^{*} \mathrm{P}<0.05$ vs. group C. " $\mathrm{P}<0.05$ vs. group $\mathrm{U}$. IL- $1 \beta$, Interleukin-1 $\beta$; TNF- $\alpha$, tumor necrosis factor $\alpha$; NF- $\mathrm{kB}$, nuclear factor $\kappa \mathrm{B} ; \mathrm{C}$, control group; $\mathrm{U}$, urethane-induced adenocarcinoma group; P, prebiotics-gavage intervention group.
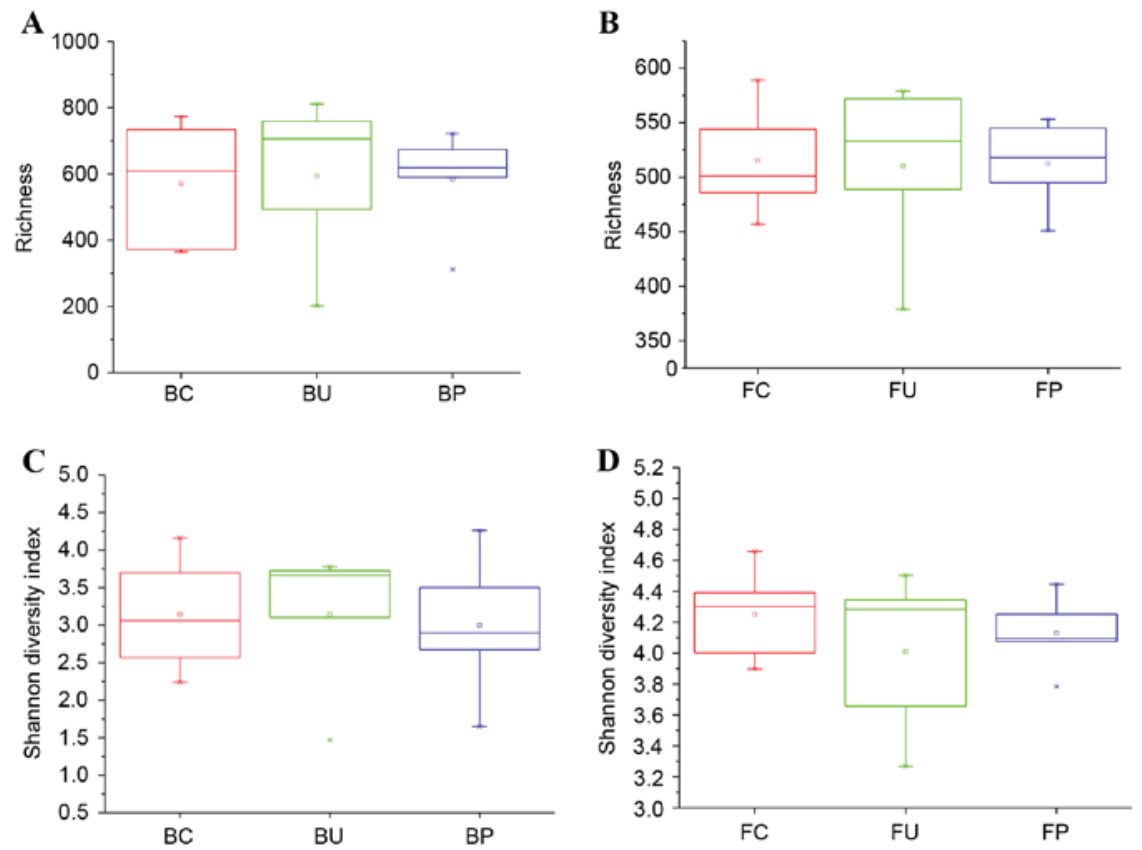

Figure 3. Boxplot demonstrating indices of the (A and B) richness and (C and D) Shannon diversity of the intestinal and respiratory microbiota. No significant differences in the richness or diversity were observed among the 3 groups. BAL, bronchoalveolar lavage; BC, microbiota from BAL in control group C; BU, microbiota from BAL in urethane-induced adenocarcinoma group U; BP, microbiota from BAL in prebiotics-gavage intervention group P; FC, microbiota from fecal matter in control group C; FU, microbiota from fecal matter in urethane-induced adenocarcinoma group U; FP, microbiota from fecal matter in prebiotics-gavage intervention group $\mathrm{P}$.

was not statistically significant (the data that exhibited significant variation is listed in Table I, and others are not shown).

Effect of prebiotics to the OTU of microbiome. Finally, the OTU was used to analyse the difference between microbiota. In the lower respiratory tract, the levels of abundance of Clostridiales, Lachnospiraceae, Pedobacter and iiil-15 families decreased in group $\mathrm{U}$ compared with group $\mathrm{C}$, and the abundance of the Clostridiales and Lachnosipraceae families became equivalent to the level in the control group in group $\mathrm{P}$ when the prebiotics were administered. The levels of abundance of S24-7 and Erythroba families increased in group U compared with group $\mathrm{C}$, and the level in group $\mathrm{P}$ became equivalent to the levels of the control group when prebiotics were administered. These data are demonstrated in Table II; other families without significant differences among groups 


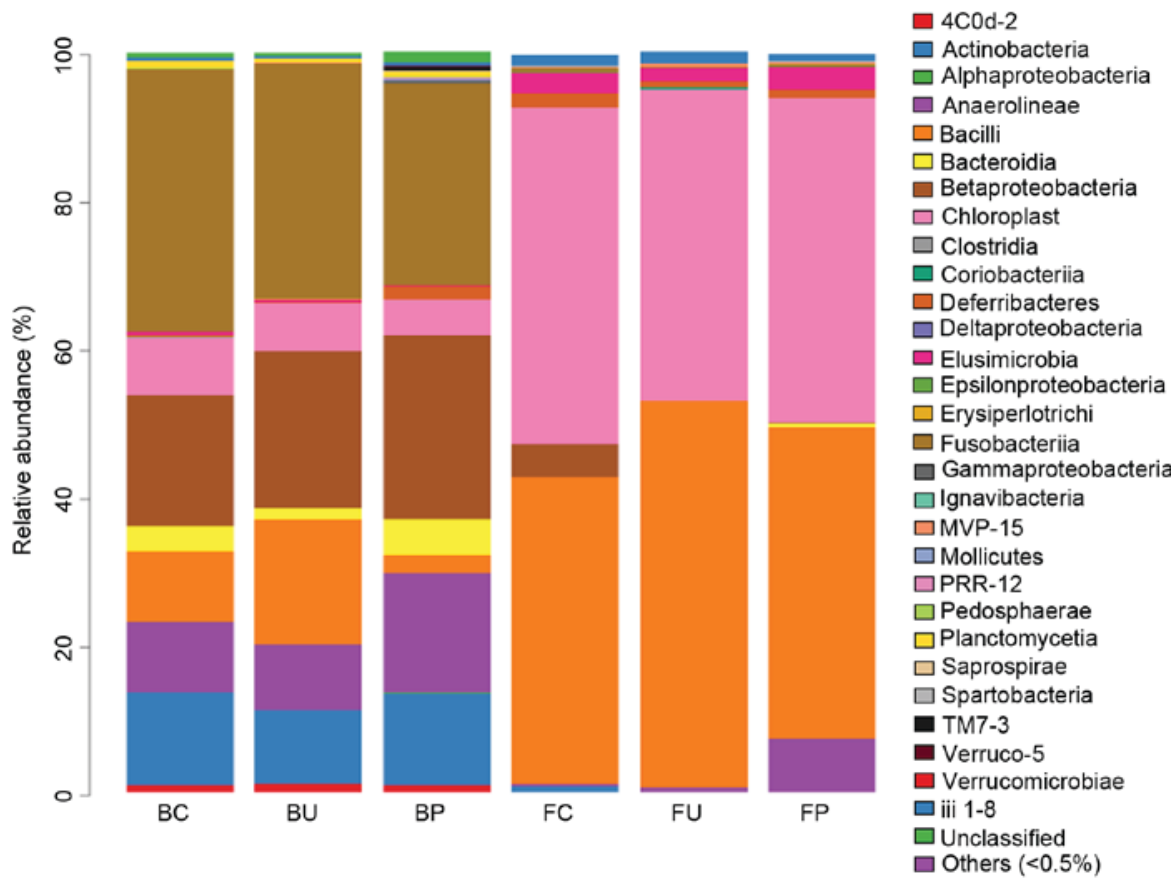

Figure 4. Microbial community comparisons and relative abundances at the class level. BAL, bronchoalveolar lavage, BC, microbiota from BAL in control group $\mathrm{C} ; \mathrm{BU}$, microbiota from $\mathrm{BAL}$ in urethane-induced adenocarcinoma group $\mathrm{U}$; BP, microbiota from BAL in prebiotics-gavage intervention group $\mathrm{P}$; FC, microbiota from fecal matter in control group C; FU, microbiota from fecal matter in urethane-induced adenocarcinoma group U; FP, microbiota from fecal matter in prebiotics-gavage intervention group P. Each bar graph demonstrates the mean abundance of 5 mice in each group.
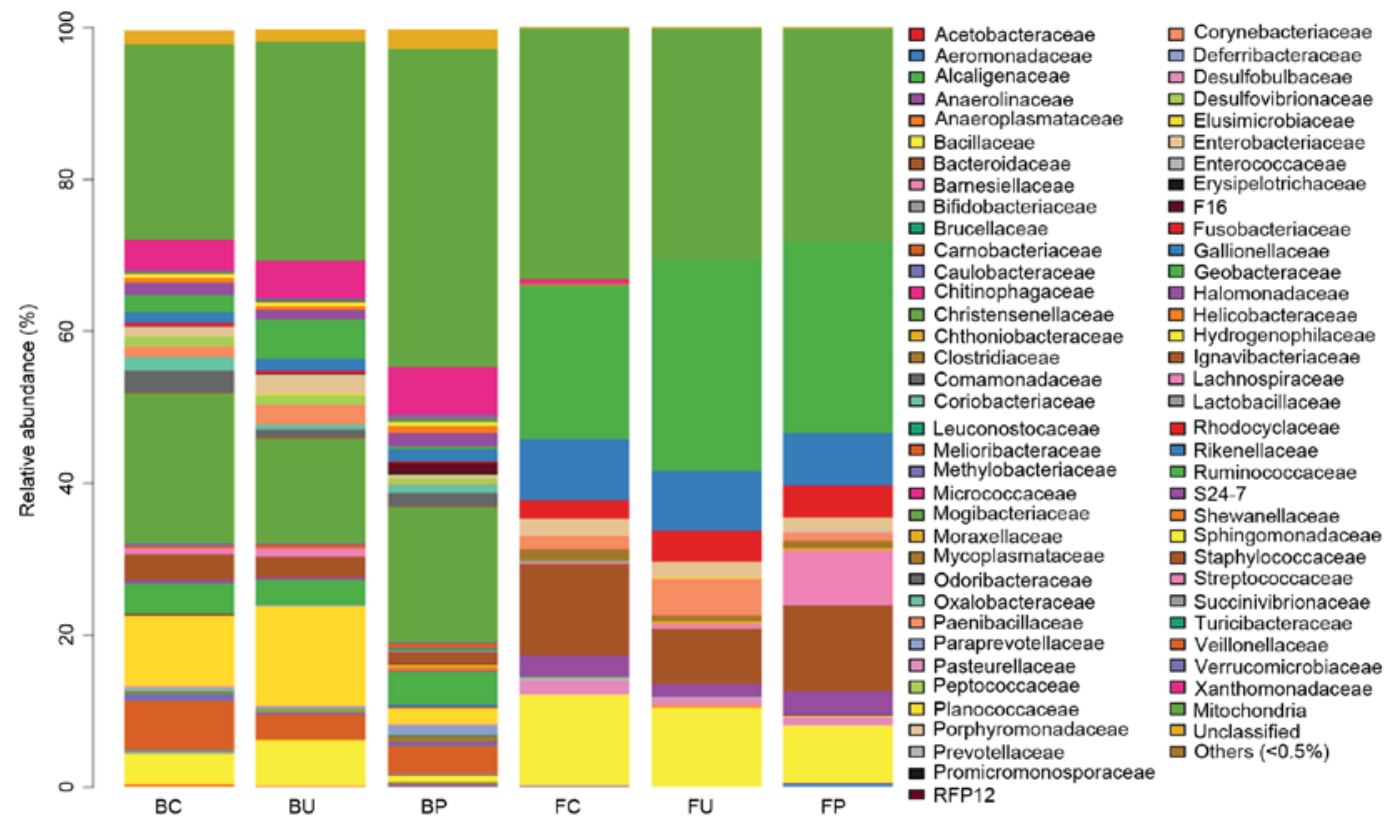

Figure 5. Microbial community comparisons and relative abundances at the Family level. BAL, bronchoalveolar lavage, BC, microbiota from BAL in control group C; BU, microbiota from BAL in urethane-induced adenocarcinoma group $\mathrm{U}$; BP, microbiota from BAL in prebiotics-gavage intervention group P; FC, microbiota from fecal matter in control group C; FU, microbiota from fecal matter in urethane-induced adenocarcinoma group U; FP, microbiota from fecal matter in prebiotics-gavage intervention group $\mathrm{P}$.

were not included due to the large number within this group In the intestinal tract, significant differences between the microbiomes of each group were observed, as illustrated in Table III. For example, the level of abundance of the S24-7, Bacteroidales and Firmicutes families increased in group $\mathrm{U}$ compared with group $\mathrm{C}$, becoming equivalent to those in the control group subsequent to the administration of prebiotics, as demonstrated in group P. The abundance levels of Clostridiales, Ruminococcus and Flexispira families exhibited a decrease in group $\mathrm{U}$ compared with group C, becoming equivalent to those in the control group subsequent to the administration of prebiotics, as demonstrated in group P. Furthermore, the Lachnospiraceae family demonstrated a similar result within the lower respiratory tract, as 
Table I. Relative abundances of bacterial families in the lower respiratory and intestinal tracts.

\begin{tabular}{lccc}
\hline & \multicolumn{3}{c}{ Relative mean abundance $(\%)$} \\
\cline { 2 - 4 } Bacterial families & $\mathrm{n}=(5)$ & $\mathrm{n}=(5)$ & $\mathrm{n}=(5)$ \\
\hline Enterobacteriaceae & $9.375(\mathrm{BC})$ & $13.042(\mathrm{BU})$ & $2.084(\mathrm{BP})^{\mathrm{c}}$ \\
Sphingomonadaceae & $0.564(\mathrm{BC})$ & $0.551(\mathrm{BU})$ & $1.059(\mathrm{BP})^{\mathrm{a}, \mathrm{c}}$ \\
Oxalobacteraceae & $0.006(\mathrm{FC})$ & $0.000(\mathrm{FU})^{\mathrm{a}}$ & $0.006(\mathrm{FP})$ \\
Paenibacillaceae & $0.002(\mathrm{FC})$ & $0.000(\mathrm{FU})^{\mathrm{b}}$ & $0.005(\mathrm{FP})$ \\
Enterobacteriaceae & $0.074(\mathrm{FC})$ & $0.016(\mathrm{FU})$ & $0.048(\mathrm{FP})^{\mathrm{c}}$ \\
Moraxellaceae & $0.188(\mathrm{FC})$ & $0.001(\mathrm{FU})$ & $0.052(\mathrm{FP})^{\mathrm{c}}$ \\
Shewanellaceae & $0.038(\mathrm{FC})$ & $0.001(\mathrm{FU})$ & $0.006(\mathrm{FP})^{\mathrm{c}}$ \\
\hline
\end{tabular}

${ }^{\mathrm{a}} \mathrm{P}<0.05$, ${ }^{\mathrm{b}} \mathrm{P}<0.01$ vs. group $\mathrm{C}$; ${ }^{\mathrm{c}} \mathrm{P}<0.05$, vs. group $\mathrm{U}$. BC, microbiota from $\mathrm{BAL}$ in control group $\mathrm{C}$; $\mathrm{BU}$, microbiota from $\mathrm{BAL}$ in urethane-induced adenocarcinoma group $\mathrm{U}$; $\mathrm{BP}$, microbiota from $\mathrm{BAL}$ in prebiotics-gavage intervention group $\mathrm{P} ; \mathrm{FC}$, microbiota from fecal matter in control group $\mathrm{C}$; FU, microbiota from fecal matter in urethane-induced adenocarcinoma group $\mathrm{U} ; \mathrm{FP}$, microbiota from fecal matter in prebiotics-gavage intervention group $\mathrm{P}$.

summarized in Table III. Other families without significant differences between the groups were not included, due to the large number within this group.

\section{Discussion}

At present, intestinal microbiota are considered to be the most important type of microbiome in humans, with known associations with numerous diseases including obesity (28), diabetes (29), inflammatory bowel disease (30) and cancer $(9,18)$. In experimental animals and humans, there is evidence to support the hypothesis that the commensal microbiome serves an important role in carcinogenesis, tumor progression and therapy (18).

The association between the intestinal microbiome, inflammation and cancer has also been widely studied. For example, a recent study concluded that the intestinal microbiome affected the intestine of the host and other organs such as the lungs, due to the circulation of metabolites produced in the intestines throughout the body (31). Multiple studies have demonstrated that the microbiome existing in the respiratory tract is associated with numerous diseases of the lungs, including COPD $(12,13)$, asthma (14) and lung cancer (15). Additionally, the microbiome of the respiratory tract serves an important role in the exacerbation of chronic lung diseases (32). Otherwise, the microbiomes of the airway and intestinal tracts may affect each other through the gut-lung axle $(23,33)$. For example, a study conducted by Madan et al (34) indicated that changes in diet resulted in an alteration of the intestinal tract microflora, and in an alteration in the respiratory tract microflora. Previous studies have demonstrated that respiratory and intestinal microbiomes are associated with inflammation, which was demonstrated to be one of the most important factors in the carcinogenesis of lung cancer $(16,17)$. Investigation into the association between microbiomes in the lower airways and
Table II. OTU of the respiratory microbiome.

Average of relative abundance in different group

\begin{tabular}{lccc}
\cline { 2 - 4 } OTU & $\mathrm{BC}(\mathrm{n}=5)$ & $\mathrm{BU}(\mathrm{n}=5)$ & $\mathrm{BP}(\mathrm{n}=5)$ \\
\hline Clostridiales & 2.8 & $0.8^{\mathrm{a}}$ & 1.2 \\
Lachnospiraceae & 2.6 & $0.0^{\mathrm{b}}$ & 0.8 \\
Pedobacter & 2.4 & $0.2^{\mathrm{b}}$ & $0.0^{\mathrm{b}}$ \\
iii1-15 & 1.2 & $0.0^{\mathrm{b}}$ & $0.0^{\mathrm{b}}$ \\
Rikenellaceae & 0.0 & $7.0^{\mathrm{b}}$ & 1.0 \\
S24-7 & 0.2 & $4.2^{\mathrm{b}}$ & 2.4 \\
Erythroba & 0.4 & 2.2 & $0^{\mathrm{c}}$ \\
\hline
\end{tabular}

${ }^{\mathrm{a}} \mathrm{P}<0.05,{ }^{\mathrm{b}} \mathrm{P}<0.001$ vs. group $\mathrm{BC}$; ${ }^{\mathrm{c}} \mathrm{P}<0.001$ vs. group $\mathrm{BU}$. $\mathrm{BC}$, microbiota from $\mathrm{BAL}$ in control group $\mathrm{C}$; $\mathrm{BU}$, microbiota from $\mathrm{BAL}$ in urethane-induced adenocarcinoma group $\mathrm{U}$; BP, microbiota from BAL in prebiotics-gavage intervention group $\mathrm{P}$; OTU, operational taxonomic units.

Table III. OTU of the intestinal microbiome.

\begin{tabular}{|c|c|c|c|}
\hline \multirow[b]{2}{*}{ Out } & \multicolumn{3}{|c|}{$\begin{array}{l}\text { Average of relative abundance } \\
\text { in different group }\end{array}$} \\
\hline & $\mathrm{FC}(\mathrm{n}=5)$ & $\mathrm{FU}(\mathrm{n}=5)$ & $\mathrm{FP}(\mathrm{n}=5)$ \\
\hline S24-7 & $1,187.5$ & $4,050.6^{\mathrm{a}}$ & $863.2^{\mathrm{d}}$ \\
\hline Clostridiales & 41.8 & $10.4^{\mathrm{a}}$ & $57.0^{\mathrm{d}}$ \\
\hline Bacteroidales & 0.0 & $0.6^{\mathrm{c}}$ & $0.0^{\mathrm{e}}$ \\
\hline Firmicutes & 1.0 & $7.2^{\mathrm{c}}$ & $0.0^{\mathrm{e}}$ \\
\hline Ruminococcus & 85 & $27.4^{\mathrm{a}}$ & 40.6 \\
\hline Ruminococcus $g n$ & 24.4 & $5^{\mathrm{b}}$ & 17.2 \\
\hline Bacteroides & 0.2 & $1.8^{\mathrm{c}}$ & 0.8 \\
\hline Flexispira & 132.6 & $0.0^{\mathrm{c}}$ & 84.0 \\
\hline Adlercreutzia & 0.0 & $1.6^{\mathrm{c}}$ & 0.6 \\
\hline Oxalobacteraceae & 1.2 & $0.0^{\mathrm{c}}$ & 1.8 \\
\hline Lachnospiraceae & 18.4 & $2.2^{\mathrm{c}}$ & 10.6 \\
\hline Desulfovibrionaceae & 35 & $0.2^{\mathrm{c}}$ & $0.0^{\mathrm{c}}$ \\
\hline Paenibacillus & 0.8 & $0.0^{\mathrm{c}}$ & 2.0 \\
\hline AF12 & 0.4 & $141.4^{\mathrm{c}}$ & $48.0^{c}$ \\
\hline Bacteria & 0 & 0 & $2.0^{\mathrm{e}}$ \\
\hline Lactobacillus & 0.4 & 0 & $3.2^{\mathrm{e}}$ \\
\hline Lactobacillus ruminis & 0.2 & 0 & $0.6^{\mathrm{e}}$ \\
\hline Lactococcus garvieae & 1.8 & 2.8 & $0.0^{\mathrm{e}}$ \\
\hline Rikenellaceae & 0 & 0 & $1.4^{\mathrm{e}}$ \\
\hline Shewanella algae & 15.4 & 0.4 & $2.4^{\mathrm{e}}$ \\
\hline Ruminococcaceae & 1.2 & 2.2 & $0.4^{\mathrm{e}}$ \\
\hline Escherichia coli & $2,850.4$ & $4,058.6$ & $510.0^{\mathrm{d}}$ \\
\hline Helicobacteraceae & 0.8 & 0.2 & $3.6^{\mathrm{e}}$ \\
\hline
\end{tabular}

${ }^{\mathrm{a}} \mathrm{P}<0.05,{ }^{\mathrm{b}} \mathrm{P}<0.01,{ }^{\mathrm{c}} \mathrm{P}<0.001$ vs. group $\mathrm{FC} ;{ }^{\mathrm{d}} \mathrm{P}<0.05,{ }^{\mathrm{e}} \mathrm{P}<0.001$ vs. group FU. FC, microbiota from feces in the control group $\mathrm{C}$; FU, microbiota from feces in the urethane-induced adenocarcinoma group U; FP, microbiota from feces in the prebiotics-gavage intervention group $\mathrm{P}$. 
the intestinal tract, inflammation and lung cancer is therefore required.

Furthermore, the development of metagenomics, including 16S rRNA gene pyrosequencing technique and bioinformatics (7) have provided an increased understanding of the role of the human microbiome in the development of cancer. The microbiome has become an important area of research. However, data associating the changes in the airway and intestinal microbiome to lung cancer remain scarce. Thus, the aim of the present study was to investigate and explain the association between microbiota, inflammation and lung cancer through a urethane-induced pulmonary adenocarcinoma mouse model, which is widely used in research in lung cancer (21) and the microbiome (35).

The present study identified no significant variation in the richness and diversity of microbiome among the 3 groups, as demonstrated in Fig. 1, which may be associated with the individual variation and the sample number of each group. However, there were some significant variations between the intestinal and the respiratory tract microbiomes at a familial level, which is demonstrated in Tables I, II and III. The levels of abundance of the S24-7, Bacteroidales and Firmicutes families were increased in the intestinal tract of urethane-induced pulmonary adenocarcinoma mice (U) compared with the control group (C), but were reduced when prebiotics were administered to the urethane-induced pulmonary adenocarcinoma mice (group P). The abundance levels of the Clostridiales family were decreased in the intestinal tract of urethane-induced pulmonary adenocarcinoma mice (U) compared with the control group (C), but were increased when prebiotics were administered to the urethane-induced pulmonary adenocarcinoma mice (group P). Additionally, the S24-7, Clostridiales and Lachnospiraceae families exhibited similar variation in the respiratory and intestinal tracts, and the variation between each group and different organ sites- the lungs and the intestine- indicated that an interaction existed between the intestinal and respiratory tract microbiomes, and prebiotics may affect these microbiomes, which was similar to data revealed in a previous study (34).

Multiple previous studies identified similar changes in the levels of abundance of the Clostridiales, Bacteroidales and S24-7 families observed in the present study. For example, Baxter et al (36) demonstrated that the number of Bacteroidales families present was associated with a higher rate of tumorigenesis, whilst the numbers of Clostridiales families present was associated with a lower rate of tumorigenesis. In the present study, the levels of abundance of the Bacteroidales and S24-7 families increased in group $\mathrm{U}$ compared with group $\mathrm{C}$ in the lower respiratory and intestinal tracts, whilst the level of abundance of the Clostridiales families decreased. These variations were also found in a study by Schwab et al (37) concerning murine microbiota activity and interactions with the host during acute inflammation and recovery. Furthermore, a reduction in the level of abundance of intestinal Clostridiales families was reported to precede the development of nosocomial Clostridium difficile infection (38), and decreased abundance in Clostridiales families was strongly correlated with inflammatory bowel disease (IBD) status (39). Whilst an increase in the relative proportion of Bacteroidales families was identified in high-fat-fed mice with high levels of inflammation (40), Bacteroidales families were also found in oral mucosae, and exhibited higher levels of abundance in recurrent aphthous stomatitis patients compared with healthy controls (41). Thus, the variations in Clostridials, Bacteroidales and S24-7 families in the lower respiratory and intestinal tracts may exert significant effects on the levels of inflammation and the carcinogenesis of lung cancer.

In the present study, the results from the analysis of the inflammatory markers revealed that the inflammation level in the urethane-induced pulmonary adenocarcinoma mice was higher than the level of the control group, which was similar to the data observed in previous studies $(21,42)$. Additionally, the level of inflammation decreased when prebiotics were administered to the group of pulmonary adenocarcinoma mice. The prebiotics used consisted of a mixture plant polysaccharides, which are utilized by the microbiome to promote the growth of certain microorganisms, particularly probiotic microorganisms such as Lactobacillus, the level of which increased in group P in the intestinal tract, as demonstrated in Table III.

In conclusion, the carcinogen urethane affected the levels of inflammation and the composition of the microbiome, and prebiotics inhibited the urethane-induced elevation of the level of inflammation in mice, possibly through the regulation of the microbiome in the intestinal and respiratory tracts, but did not inhibit the development of pulmonary adenocarcinoma that was possibly associated with the interference time and the concentration of the prebiotics. Additional studies are required to investigate these issues. In the present study, it was demonstrated that prebiotics may increase levels of inflammation and the composition of the microbiome in the intestinal and respiratory tracts, and improve the treatment of lung cancer. Understanding the role of the intestinal and respiratory microbiomes is important, in order to identify novel targets and approaches for improving the treatment and diagnosis of lung cancer.

\section{Acknowledgements}

The authors would like to thank Professor Yiming Lu (Beijing Institute of Radiation Medicine, State Key Laboratory of Proteomics, Cognitive and Mental Health Research Center of The PLA, Beijing, China) for technical assistance with $16 \mathrm{~S}$ rRNA sequence raw data processing.

\section{Funding}

The present study was supported by the National Natural Science Foundation of China (grant nos. 82171206 and 31401141) and the Foundation of the Center of Translational Medicine between Beijing Proteome Research Center and Baodi Clinical Institute of Tianjin medical university (grant no. TMRC201301).

\section{Availability of data and materials}

The datasets used and analyzed during the present study are available from the corresponding author on reasonable request.

\section{Authors' contributions}

CZ and GW conceived and designed the study. ZD, ZL, CS and HX performed the animal experiments, sera preparation and DNA extraction. ZD and CZ performed $16 \mathrm{~S}$ data generation and analysis. ZC, JL and HW conducted pathological analysis. 
ZD, ZL, CZ and GW participated in the design of the study and drafted the manuscript.

\section{Ethics approval and consent to participate}

All mouse experiments were performed in accordance with the approved guidelines of the Academy of Military Medical Sciences. The experimental protocol was approved by the Ethics Committee for Animal Experimentation of the Academy of Military Medical Sciences.

\section{Consent for publication}

Not applicable.

\section{Competing interests}

The authors declare that they have no competing interests.

\section{References}

1. Torre LA, Bray F, Siegel RL, Ferlay J, Lortet-Tieulent J and Jemal A: Global cancer statistics, 2012. CA Cancer J Clin 65: 87-108, 2015

2. DeSantis CE, Lin CC, Mariotto AB, Siegel RL, Stein KD, Kramer JL, Alteri R, Robbins AS and Jemal A: Cancer treatment and survivorship statistics, 2014. CA Cancer J Clin 64: 252-271, 2014.

3. Kamada N, Seo SU, Chen GY and Nunez G: Role of the gut microbiota in immunity and inflammatory disease. Nat Rev Immunol 13: 321-335, 2013.

4. Seoane A, Bessa X, Balleste B, O'Callaghan E, Panadès A, Alameda F, Navarro S, Gallén M, Andreu M and Bory F: Helicobacter pylori and gastric cancer: Relationship with histological subtype and tumor location. Gastroenterol Hepatol 28 60-64, 2005 (In Spanish).

5. Smith C: EBV and nasopharyngeal carcinoma: A target for cellular therapies. Immunotherapy 5: 821-824, 2013.

6. Lynge E and Rebolj M: Primary HPV screening for cervical cancer prevention: Results from European trials. Nat Rev Clin Oncol 6: 699-706, 2009.

7. Martin R, Miquel S, Langella P and Bermudez-Humaran LG: The role of metagenomics in understanding the human microbiome in health and disease. Virulence 5: 413-423, 2014.

8. Schmidt BL, Kuczynski J, Bhattacharya A, Huey B, Corby PM, Queiroz EL, Nightingale K, Kerr AR, DeLacure MD Veeramachaneni R, et al: Changes in abundance of oral microbiota associated with oral cancer. PLoS One 9: e98741, 2014.

9. Farrell JJ, Zhang L, Zhou H, Chia D, Elashoff D, Akin D, Paster BJ, Joshipura K and Wong DT: Variations of oral microbiota are associated with pancreatic diseases including pancreatic cancer. Gut 61: 582-588, 2012.

10. Dejea CM, Wick EC, Hechenbleikner EM, White JR, Mark Welch JL, Rossetti BJ, Peterson SN, Snesrud EC, Borisy GG, Lazarev M, et al: Microbiota organization is a distinct feature of proximal colorectal cancers. Proc Natl Acad Sci USA 111: 18321-18326, 2014.

11. Zeller G, Tap J, Voigt AY, Sunagawa S, Kultima JR, Costea PI, Amiot A, Böhm J, Brunetti F, Habermann N, et al: Potential of fecal microbiota for early-stage detection of colorectal cancer. Mol Syst Biol 10: 766, 2014.

12. Han MK, Huang YJ, Lipuma JJ, Boushey HA, Boucher RC, Cookson WO, Curtis JL, Erb-Downward J, Lynch SV, Sethi S, et al: Significance of the microbiome in obstructive lung disease. Thorax 67: 456-463, 2012.

13. Garcia-Nuñez M, Millares L, Pomares X, Ferrari R, Pérez-Brocal V, Gallego M, Espasa M, Moya A and Monsó E: Severity-related changes of bronchial microbiome in chronic obstructive pulmonary disease. J Clin Microbiol 52: 4217-4223, 2014.

14. Gurwitz D and Lunshof JE: Farm microbiome and childhood asthma. N Engl J Med 364: 1972, 2011.
15. Hosgood HD III, Sapkota AR, Rothman N, Rohan T, Hu W, Xu J, Vermeulen R, He X, White JR, Wu G, et al: The potential role of lung microbiota in lung cancer attributed to household coal burning exposures. Environ Mol Mutagen 55: 643-651, 2014.

16. Stathopoulos GT, Sherrill TP, Cheng DS, Scoggins RM, Han W, Polosukhin VV, Connelly L, Yull FE, Fingleton B and Blackwell TS: Epithelial NF-kappa B activation promotes urethane-induced lung carcinogenesis. Proc Natl Acad Sci USA 104: 18514-18519, 2007.

17. Malkinson AM: Role of inflammation in mouse lung tumorigenesis: A review. Exp Lung Res 31: 57-82, 2005.

18. Dzutsev A, Goldszmid RS, Viaud S, Zitvogel L and Trinchieri G: The role of the microbiota in inflammation, carcinogenesis, and cancer therapy. Eur J Immunol 45: 17-31, 2015.

19. Belkaid Y and Hand TW: Role of the microbiota in immunity and inflammation. Cell 157: 121-141, 2014.

20. de Martel C, Ferlay J, Franceschi S, Vignat J, Bray F, Forman D and Plummer M: Global burden of cancers attributable to infections in 2008: A review and synthetic analysis. Lancet Oncol 13: 607-615, 2012.

21. Narayan C and Kumar A: Constitutive over expression of IL-1 $\beta$, IL-6, NF- $\mathrm{KB}$, and Stat 3 is a potential cause of lung tumorgenesis in urethane (ethyl carbamate) induced Balb/c mice. J Carcinog 11: 9,2012.

22. Ihara S, Kida H, Arase H, Tripathi LP, Chen YA, Kimura T, Yoshida M, Kashiwa Y, Hirata H, Fukamizu R, et al: Inhibitory roles of signal transducer and activator of transcription 3 in antitumor immunity during carcinogen-induced lung tumorigenesis. Cancer Res 72: 2990-2999, 2012.

23. Barfod KK, Roggenbuck M, Hansen LH, Schjorring S, Larsen ST, Sorensen SJ and Krogfelt KA: The murine lung microbiome in relation to the intestinal and vaginal bacterial communities. BMC Microbiol 13: 303, 2013.

24. Zhou D, Zhang H, Bai Z, Zhang A, Bai F, Luo X, Hou Y, Ding X, Sun B, Sun X, et al: Exposure to soil, house dust and decaying plants increases gut microbial diversity and decreases serum immunoglobulin E levels in BALB/c mice. Environ Microbiol 18: 1326-1337, 2015.

25. Edgar RC: UPARSE: Highly accurate OTU sequences from microbial amplicon reads. Nat Methods 10: 996-998, 2013.

26. Edgar RC, Haas BJ, Clemente JC, Quince C and Knight R: UCHIME improves sensitivity and speed of chimera detection. Bioinformatics 27: 2194-2200, 2011.

27. Rodrigues NF, Kästle J, Coutinho TJ, Amorim AT, Campos GB, Santos VM, Marques LM, Timenetsky J and de Farias ST: Qualitative analysis of the vaginal microbiota of healthy cattle and cattle with genital-tract disease. Genet Mol Res 14: 6518-6528, 2015.

28. Ferolla SM, Armiliato GN, Couto CA and Ferrari TC: The role of intestinal bacteria overgrowth in obesity-related nonalcoholic fatty liver disease. Nutrients 6: 5583-5599, 2014.

29. Karlsson F, Tremaroli V, Nielsen J and Backhed F: Assessing the Human Gut Microbiota in Metabolic Diseases. Diabetes 62: 3341-3349, 2013.

30. Tong M, Li X, Wegener Parfrey L, Roth B, Ippoliti A, Wei B, Borneman J, McGovern DP, Frank DN, Li E, et al: A modular organization of the human intestinal mucosal microbiota and its association with inflammatory bowel disease. PLoS One 8: e80702, 2013.

31. Ohtani N: Microbiome and cancer. Semin Immunopathol 37: 65-72, 2015.

32. Dickson RP, Martinez FJ and Huffnagle GB: The role of the microbiome in exacerbations of chronic lung diseases. Lancet 384: 691-702, 2014.

33. Sze MA, Tsuruta M, Yang SW, Oh Y, Man SF, Hogg JC and Sin DD: Changes in the bacterial microbiota in gut, blood, and lungs following acute LPS instillation into mice lungs. PLoS One 9: e111228, 2014.

34. Madan JC, Koestler DC, Stanton BA, Davidson L, Moulton LA, Housman ML, Moore JH, Guill MF, Morrison HG, Sogin ML, et al: Serial analysis of the gut and respiratory microbiome in cystic fibrosis in infancy: Interaction between intestinal and respiratory tracts and impact of nutritional exposures. MBio 3: e00251-12, 2012.

35. Garzoni C, Brugger SD, Qi W, Wasmer S, Cusini A, Dumont P, Gorgievski-Hrisoho M, Mühlemann K, von Garnier C and Hilty M: Microbial communities in the respiratory tract of patients with interstitial lung disease. Thorax 68: 1150-6, 2013.

36. Baxter NT, Zackular JP, Chen GY and Schloss PD: Structure of the gut microbiome following colonization with human feces determines colonic tumor burden. Microbiome 2: 20, 2014. 
37. Schwab C, Berry D, Rauch I, Rennisch I, Ramesmayer J, Hainzl E, Heider S, Decker T, Kenner L, Müller M, et al: Longitudinal study of murine microbiota activity and interactions with the host during acute inflammation and recovery. ISME J 8: 1101-1114, 2014.

38. Vincent C, Stephens DA, Loo VG, Edens TJ, Behr MA, Dewar K and Manges AR: Reductions in intestinal Clostridiales precede the development of nosocomial Clostridium difficile infection. Microbiome 1: 18, 2013.

39. Gevers D, Kugathasan S, Denson LA, Vázquez-Baeza Y, Van Treuren W, Ren B, Schwager E, Knights D, Song SJ, Yassour M, et al: The treatment-naive microbiome in new-onset Crohn's disease. Cell Host Microbe 15: 382-392, 2014.

40. de La Serre CB, Ellis CL, Lee J, Hartman AL, Rutledge JC and Raybould HE: Propensity to high-fat diet-induced obesity in rats is associated with changes in the gut microbiota and gut inflammation. Am J Physiol Gastrointest Liver Physiol 299: G440-G448, 2010.
41. Hijazi K, Lowe T, Meharg C, Berry SH, Foley J and Hold GL: Mucosal Microbiome in Patients with Recurrent Aphthous Stomatitis. J Dent Res 94: 87, 2015S-94S.

42. Jan Treda C, Fukuhara T, Suzuki T, Nakamura A, Zaini J, Kikuchi T, Ebina M and Nukiwa T: Secretory leukocyte protease inhibitor modulates urethane-induced lung carcinogenesis. Carcinogenesis 35: 896, 2014.

This work is licensed under a Creative Commons Attribution-NonCommercial-NoDerivatives 4.0 International (CC BY-NC-ND 4.0) License. 